\title{
A TRANSPORTABLE REFRACTOMETER FOR ASSESSMENT OF PRES- SURE IN THE KPA RANGE WITH PPM LEVEL PRECISION
}

\author{
C. Forssén ${ }^{1,2}$, I. Silander ${ }^{1}$, D. Szabo ${ }^{2}$, G. Jönsson ${ }^{2}$, M. Bjerling 2 , T. Hausmaninger ${ }^{3}$, O. Axner ${ }^{1}$, M. Zelan ${ }^{2}$ \\ ${ }^{1}$ Department of Physics, Umeå University, Sweden, \\ clayton.forssen@umu.se, isak.silander@umu.se, ove.axner@umu.se \\ ${ }^{2}$ Measurement Technology, RISE Research Institutes of Sweden, Borås, Sweden, \\ david.szabo@ri.se, gustav.jonsson@ri.se, martin.bjerling@ @ri.se, martin.zelan@ri.se \\ ${ }^{3}$ National Metrology Institute VTT MIKES, Tekniikantie, Finland, thomas.hausmaninger@vtt.fi
}

\begin{abstract}
:
A transportable refractometer for assessment of $\mathrm{kPa}$ pressures with ppm level precision is presented. It is based on the GAs MOdulation Refractometry (GAMOR) methodology, making it resistant to fluctuations and drifts. At the National Metrology Institute at RISE, Sweden, the system assessed pressures in the $4.3-8.7 \mathrm{kPa}$ range with sub-ppm precision $(0.5-0.9 \mathrm{ppm})$. The system was thereafter disassembled, packed, and transported $1040 \mathrm{~km}$ to Umeå University, where it, after unpacking and reassembling, demonstrated a similar precision (0.8 - 2.1 ppm). This shows that the system can be disassembled, packed, transported, unpacked, and reassembled with virtually unchanged performance.
\end{abstract}

Keywords: Refractometry; Pressure; GAMOR; Transportable

\section{INTRODUCTION}

Good knowledge of gas abundance (molar density as well as pressure) is of importance in a variety of scientific situations and industrial processes, from basic and applied research (including laser spectroscopy) to process monitoring and feedback control in industry. For this, reliable measurement systems or gauges, traceable to primary standards, that can assess molar density or pressure, are needed.

In the SI-system of units, the realization of the Pascal relies on mechanical primary devices such as pressure balances and liquid manometers that realize pressure as a force per area [1-2]. Although current conventional techniques demonstrate excellent performance under optimal conditions and handling $[1,3,4]$, their performance have remained nearly unchanged for decades. They are often limited in terms of working range, in particular pressure balances, which have a lower limit given by the mass of the piston, and they need to be firmly stationed in a highly stabilized laboratory, requiring either the system to be characterized to be transported or the use of transportable secondary standards to calibrate systems out in scientific laboratories or industrial facilities.

An alternative path to realize pressure of a gas is by the use of an equation of state, in which pressure is given as a function of density and temperature [5, $6]$, and the density is related to refractivity by the Lorentz-Lorenz equation [7-9]. This, in combination with the revision of the SI-system [10], in which the Boltzmann constant is fixed, allows for a primary standard for pressure in terms of refractivity and temperature [6].

Although there are a variety of means to assess refractivity [11-15], the most sensitive realizations are refractometry based on a Fabry-Pérot (FP) cavity $[11,16-17]$. However, ordinary FP-based refractometers are often limited by the stability of the length of the cavity $[5,18,19]$. Hence an exceptionally good mechanical and thermal stability of the system is required.

To remedy this, a novel refractometric methodology, based on gas modulation, referred to as GAMOR (GAs MOdulation Refractometry), has recently been developed [20-21]. Correctly done, it can provide pressure assessments that are insensitive to both length drifts (and relaxations) of the cavity spacer as well as gas leakages and outgassing in the reference cavity $[20,22]$. Furthermore, it has the potential to provide a dynamic range of at least 8 orders of magnitude, i.e. from the low $\mathrm{mPa}$ region to atmospheric pressures and above.

These properties make a refractometer system based on the GAMOR methodology ideal for use outside well-controlled laboratories, including industrial environments, and thereby also suitable for transportation. This implies that, whenever characterized, and when shown to retain the characterization during transportation, a GAMOR-based refractometry system can be used as a travelling standard 
by which other pressure measuring systems directly can be characterized.

Even if not properly characterized, due to its high precision and large linear dynamic range, such a transportable system can be used in combination with a local primary standard for calibration / characterization of other pressure systems, including those that do not have a measurement range that overlap with the local primary standard.

This work presents a transportable refractometer for assessment of pressure in the $\mathrm{kPa}$ range with ppm level precision. It describes the instrumentation and the experimental method used in some detail, summarizes the results from a successful transportation, identifies shortcomings of the system, and comments on upgrades that are currently being implemented.

\section{THEORY}

The GAMOR-methodology is described in detail elsewhere where extensive descriptions and explanations of its theory, procedures, and abilities of the method are given [20-22].

In short, the refractivity of the gas let into the measurement cavity, $n-1$, can be expressed as

$$
n-1=\frac{\overline{\Delta f}+\overline{\Delta q}}{1-\overline{\Delta f}+\varepsilon} \text {. }
$$

Here $\overline{\Delta f}=\Delta f / v_{0}$, where, $\Delta f$ is the shift in the beat frequency between the two laser fields when the measurement cavity, after being evacuated, is filled with the gas to be characterized, formally given by $f_{g}-f_{0}$ where $f_{g}$ and $f_{0}$ are the filled and evacuated measurement cavity beat frequencies respectively, while $v_{0}$ is the frequency of the cavity mode addressed in the empty measurement cavity (denoted $q_{0}$ ). $\overline{\Delta q}$ represents $\Delta q / q_{0}$, where $\Delta q$ is the number of modes the measurement laser jumps when the measurement cavity is filled with gas. $\varepsilon$ is the refractivity-normalized deformation coefficient of the measurement cavity, defined by $\varepsilon(n-1)=\delta L / L_{0}$, where $\delta L$ is its change in length when it is filled with gas (which includes effects both from an altered length of the cavity spacer and distortion of the mirrors), while $L_{0}$ is its length when being empty. ${ }^{1}$ The conversion of a given refractivity, $n-1$, to gas density, $\rho$, is being performed through the extended Lorentz-Lorenz equation, which implies that the density can be assessed from the refractivity by

$$
\rho=\frac{2}{3 A_{R}}(n-1)\left[1+b_{n-1}(n-1)\right],
$$

where $A_{R}$ and $b_{n-1}$ are the molar dynamic polarizability and a series expansion coefficient, respectively, where the latter is given by $-\left(1+4 B_{R} / A_{R}^{2}\right) / 6$, where, in turn, $B_{R}$ is the second refractivity virial coefficient in the Lorentz-Lorenz equation $[7,20]$.

The corresponding pressure of the gas, $P$, can thereafter be obtained from the density as

$$
P=R T \rho\left[1+B_{\rho}(T) \rho\right],
$$

where $R$ is the ideal gas constant, $T$ is the temperature of the gas, and $B_{\rho}(T)$ is the second density virial coefficient [5].

\section{EXPERIMENTAL SETUP AND METHOD}

\subsection{Experimental setup}

The transportable refractometer is based on the GAMOR system described in Ref. [20]. As is shown in Fig. 1, it can schematically be divided into six modules, designed to allow for easy packing, transportation, and installation.

The central part of the instrumentation, the gas assessment module, module 1 in the figure, includes the DFPC which consists of two cavities drilled in a $190 \mathrm{~mm}$ long Zerodur block with a $10 \mathrm{~cm}^{2}$ square cross section $(190 \times 100 \times 100 \mathrm{~mm})$. One of the cavities acts as the reference cavity while the other serves as the measurement cavity. The cavities are made of high reflective (>99.97\%) concave mirrors with a radius of curvature of $500 \mathrm{~mm}$ attached to the Zerodur spacer by the use of a surrounding aluminum frame, yielding a finesse of $\sim 10000$ and a free spectral range (FSR) of $\sim 800 \mathrm{MHz}$. To maintain a temperature stability of $1 \mathrm{mK}$, the cavity spacer is placed inside an aluminum enclosure whose temperature is controlled by four Peltier elements.

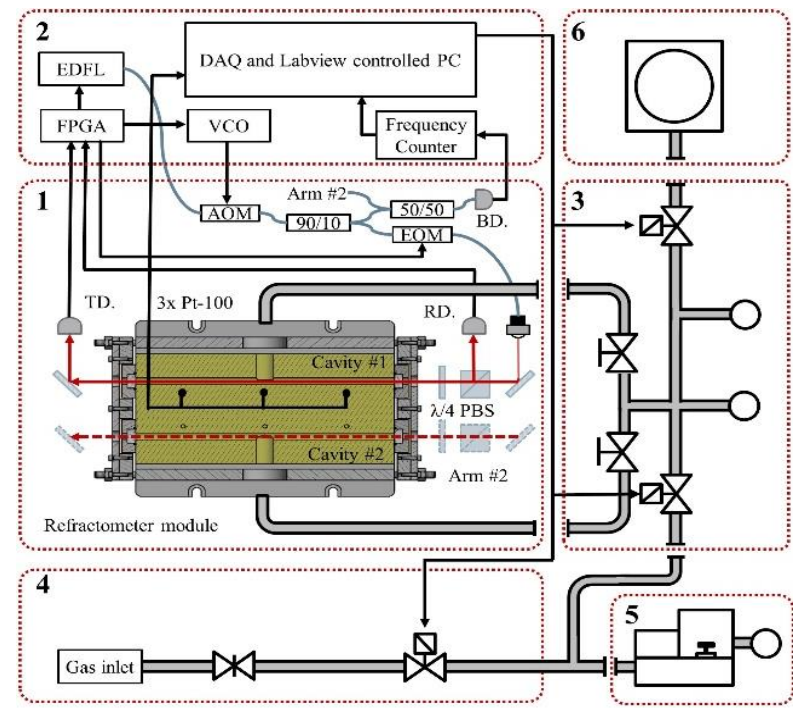

Figure 1: Schematic illustration of the experimental setup organized in separate modules; Module 1: DFPC block

\footnotetext{
${ }^{1}$ Note that no knowledge of the length of the empty cavity is needed since it is only the change in optical length of the cavity between an empty and a filled cavity that is needed for the assessment of pressure.
} 
together with the optical, acousto-optical, and electro optical components; Module 2: Electronic control and data acquisition system; Module 3: Gas distribution system; Module 4: Gas inlet system; Module 5: The device under test (DUT), represented by a pressure balance; Module 6: Gas evacuation system.

To assess the frequency of the cavity modes, the system encompasses two Er-doped fiber lasers, emitting light at $1.53 \mu \mathrm{m}$, that are locked to longitudinal modes of the cavities using the Pound-DreverHall (PDH) technique [23]. The light from the two lasers are combined in a fiber coupler and sent to a high-bandwidth photo detector, which detects their beat frequency.

As is shown in Fig. 2, all parts in module 1 are placed on an enclosed optical breadboard that easily can be attached and detached from the other modules. This allows the DFPC, which together with the optical and electro-optical components are the central and most sensitive parts of the system, to be packed and shipped as one congregated unit.

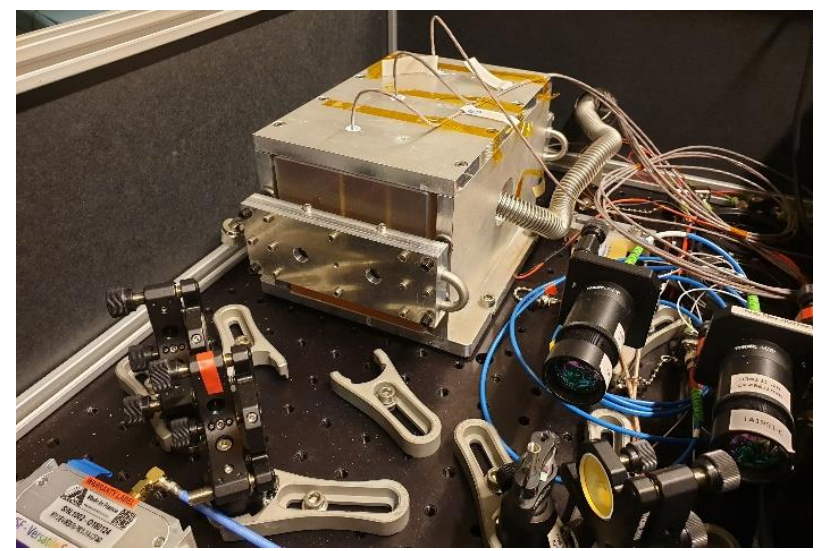

Figure 2: Picture of module 1. The Zerodur spacer is placed inside a temperature stabilized aluminum frame that also holds the cavity mirrors in place. The temperature probes were placed in holes bored in the spacer.

The main parts of the vacuum system, which constitute module 3 , comprise valves and pressure gauges that are mounted on a separate optical breadboard for easy transportation.

Module 3 is connected to, in addition to the DFPC, the modules $4-6^{2}$. Module 4 represents the gas inlet system, which consists of a needle valve to set a suitable flow and a solenoid valve connected to a locally available $\mathrm{N}_{2}$ supply (at either RISE or UmU). Module 5 comprises, at both sites, the device under testing (DUT), in this case a pressure balance (RUSKA 2465-754). Module 6 represents the gas evacuation system, here a turbo molecular pump.

Module 2 comprises electronic control and a data acquisition system. The control unit is a standard

\footnotetext{
2 The modules 4-6 are not necessarily parts of the transportable system and can be chosen depending on what is available at different sites.

${ }^{3}$ The total time for each cycle and the times for each state also depend on the capacity of the gas supply and the vacuum system. In this work the times were chosen to ensure sufficient time for both the stabilization
}

laptop running custom-made LabVIEW software that measures and regulates the cavity spacer temperature, controls the valves, and collects the beat frequency. The temperature is measured with a data acquisition module and feedback is applied to the Peltier elements. The valves are controlled through a digital output module and the beat frequency is collected from the frequency counter through a USB interface. The LabVIEW panel displays a data overview, performs real-time preliminary data analysis, and saves all data to a binary file. The final data analysis is thoroughly performed using MATLAB.

\subsection{Experimental method}

The transportable refractometer utilizes the GAMOR-methodology, which is described in detail elsewhere [20]. In short, it comprises a number of recurrent measurement cycles that each provides an independent measurement point. At the end of each cycle, to acquire a zero-pressure reference point, the measurement cavity is evacuated. The measurement cycle, which is divided into two states whose lengths were chosen to be suitable for the DUT, was taken as $200 \mathrm{~s}$. The first, representing the gas filling and stabilization state, lasted $180 \mathrm{~s}$, while the second, which encompassed gas evacuation, was maintained for $20 \mathrm{~s}^{3}$. The lengths of the filling and stabilization states were found to be sufficient for the gas in the FP-cavity to thermalize with the cavity walls.

The DUT, which also provided a stable pressure, was, in this work, at both sites, represented by a dead-weight pressure balance. For each cycle, during the evacuation state, the piston in the pressure balance drops to its resting position and consequentially resets for each measurement point ${ }^{4}$.

\subsection{Transportation of the system}

The modules are constructed so they can be packed and shipped individually (depending on what is available at the host laboratory). To assess to which extent the performance of the system (primarily its precision) is affected by disassembling, packaging, transportation (in this case withstand a rugged road-haulage), unpacking, and reassembling it, measurements were carried out at three different set pressures, both before and after the transportation. To accomplish this, five of the modules (all modules except module 5, the DUT) were packed and shipped together in a custom-built wooden box (see Fig. 3). The transportation was performed during sub $0^{\circ} \mathrm{C}$ temperatures, partly on uneven Nordic winter-roads, and carried out with no extra care taken to protect the equipment.

of the pressure balance and evacuation of the gas. The procedure was thus not optimized for speed.

${ }^{4}$ In practice, the DUT can be separated from the refractometer and the pressure can be assessed using a suitable high-performance differential pressure measurement instrument. 
Unpacking and reassembling of the system (including minor optical alignments) took approximately 4 hours. However, to minimize the amount of contaminations in the system from its exposure to atmosphere during the transportation, the system was thereafter continuously pumped down for 24 hours before any measurements were attempted.

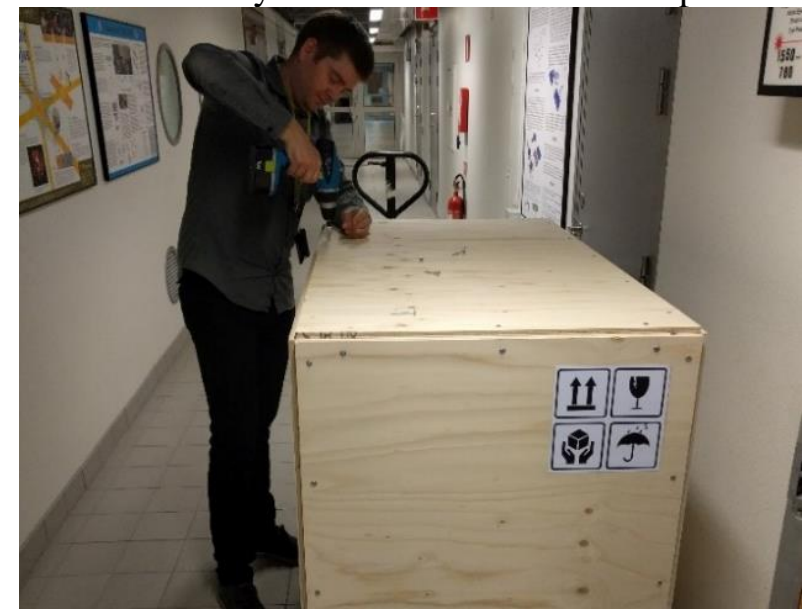

Figure 3: Picture of the box in which the refractometer was transported. The picture also manages to capture a rare proof of a last author performing practical work.

\section{RESULTS}

\subsection{Precision}

A set of measurements, comprising three measurement series, representing three different set-pressures of the pressure balance, was first performed at RISE before the system was transported to UmU, where a similar set of measurements was performed.

Since dissimilar pressure balances were used at the two premises, the set-pressures at the two premises were slightly dissimilar. At RISE they were 4303.36(63), 5766.47(67), and 8691.73(76) Pa, whereas at UmU, they were $4306.60,5769.79$, and 8695.23 Pa. The pressure balance at RISE is, by calibration, traceable to the SI-system, and provides, according to the CMC values of RISE, a pressure dependent uncertainty of $0.5+30 \times 10^{-6} p \mathrm{~Pa}(\mathrm{k}=$ 2). ${ }^{5}$ Although expected to have a similar performance, the pressure balance at UmU was last calibrated in 2010 , whereby no trustworthy uncertainty can be assigned for this work.

In order to evaluate performance in terms of precision of the transportable system, a two-hour window of each measurement series was selected by the criteria that a steady-state of the system had been reached, indicated by a stable reading of the mean of the three temperature sensors $( \pm 1 \mathrm{mK}){ }^{6}$ The selected data for the six measurement series are shown in Fig. 4 with their corresponding standard deviations, which for the three set-pressures (ca. 4300,

\footnotetext{
${ }^{5}$ Data taken from https://kcdb.bipm.org/appendixC/M/SE/M_SE.pdf.

6 The difference in time it took to reach a steady temperature state at RISE and UmU can be attributed to a difference in performance in the climate control of the laboratories.
}

5775, and $8700 \mathrm{~Pa})$ were assessed to $3.7 \mathrm{mPa}(0.9$ ppm), $5.1 \mathrm{mPa}(0.9 \mathrm{ppm})$, and $4.3 \mathrm{mPa}(0.5 \mathrm{ppm})$ for the measurements performed at RISE, and 4.1 $\mathrm{mPa}(1.0 \mathrm{ppm}), 12 \mathrm{mPa}(2.1 \mathrm{ppm})$, and $7.3 \mathrm{mPa}(0.8$ ppm) for those made at UmU, respectively. This shows that the system can be disassembled, packed,

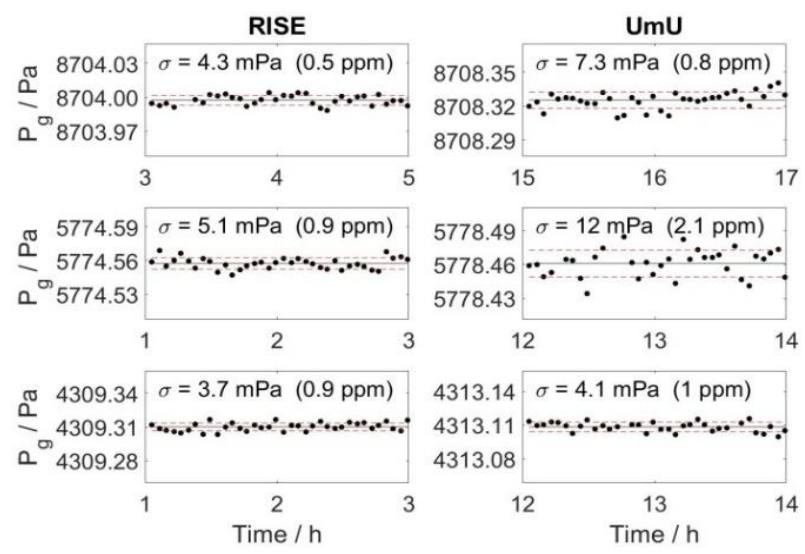

Figure 4: Two-hour windows of data selected from three measurement series performed at each location. Each dot represents an independent measurement.

transported, unpacked, and reassembled with a remained precision at the sub- or low ppm-level. ${ }^{7}$

\subsection{Linearity}

It is of interest to investigate the linearity of the system and to assess to which extent this can be affected by transportation. Figure 5 shows, by the individual markers, the assessed values of each measurement cycle (taken under quiescent conditions with the deformation coefficient, $\varepsilon$, being zero) as a function of set pressure of the pressure balance (black points, data taken at RISE; red points, data from UmU). In the main window in panel (a), all data points from a given set value $(4300,5775$, and $8700 \mathrm{~Pa})$ are, with the scale used, overlapping within the size of the markers. The inset displays an enlargement of a fraction of the data in the main window. This shows that, even on this scale, all data points taken at each of the premises still merge to a single data point, indicating the high precision of the system.

A scrutiny of the linearity of the system was performed both before and after transportation. Linear functions of the type $y=k x+m$ were fitted to the data both before and after the transportation. The deviations of the various data points with respect to the fits are displayed in panel (b). Since these deviations are smaller than the uncertainties of the pressure assessments provided by the DUTs, this indicates that the linearity of the system (in the pressure range investigated here, i.e. $4.3-8.7 \mathrm{kPa}$ ) was not compromised by the transportation.

\footnotetext{
${ }^{7}$ The slightly higher values of the standard deviation of the assessments at UmU are presumed to originate from differences in laboratory conditions, predominantly drifts of the surrounding temperature.
} 


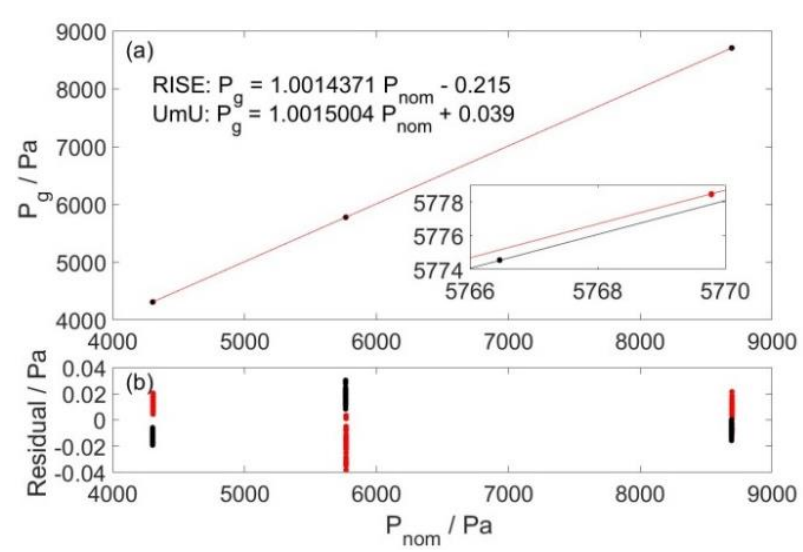

Figure 5: Panel (a): Assessed pressure by the refractometer plotted versus the nominal value of the pressure balance, (RISE: Black, UmU: Red). Solid lines: linear fits to the data from each measurement location. Inset: A three orders-of-magnitude zoom of the data around $5768 \mathrm{~Pa}$. Panel (b): The residuals to the fit.

\subsection{Accuracy}

Since the assessments presented here consistently were performed with no account taken regarding the pressure-induced cavity deformation (i.e. with $\varepsilon=0$ ), it is expected that the slopes of the fits in Fig. 5 should deviate from unity. The results also show this. For the data taken at RISE, $k=$ $1.0014371(8)$ and $m=-0.215(5)$, while for the data taken at UmU, $k=1.0015004(9)$ and $m=0.039(5)$.

The average deviation from unity of the two $k$ parameters is $0.1469 \%$ while their difference is only $63 \mathrm{ppm}$. The major reason for the large average deviation of the response comes from the fact that the deformation was not considered. Other possible reasons are uncertainties in the temperature determination of the gas, the molecular polarizability, $A_{R}$ , the local gravity, and the gas purity.

The fact that the pressure independent offsets of the fits were found to be -0.215 and $0.039 \mathrm{~Pa}$, which agree well with the uncertainties of the pressure balances, which amount to $0.5 \mathrm{~Pa}^{8}$.

\section{OUTLOOK AND RECENT UPGRADES}

Since the measurements in this work were made, we have implemented some upgrades. The system has been made more compact. The vacuum system has been upgraded with components utilizing 1/4" Swagelok tubes and moved to module 1, electronics have been custom built in a much more compact format, fiber optics have been moved to a separate module together with the AOMs and EOMs, all placed on a single $300 \times 450 \mathrm{~mm}$ breadboard. The Zerodur spacer has been replaced with an Invar cavity spacer for better thermal stabilization [24, 25].

All these upgrades have enabled the possibility of placing the entire system in a single rack cabinet.
Hence, we are presently conducting a project with the goal of being able to ship the complete system without any need of disassembly or reassembly, so as to further simplify transportation and setup. The idea is that the system simply needs to be connected to a gas supply, vacuum and a DUT at a site on which the system arrives. Furthermore, a promising procedure for characterizing a cavity (in terms of deformation, $\varepsilon$ ) has been developed [26].

\section{CONLCUSION}

A first version of a transportable refractometer for pressure assessment is presented. The refractometer was constructed around robust and exchangeable modules, which allows for easy transportation as well as a choice of different types of equipment depending on availability at various premises (e.g. pressure balances, vacuum pumps, and gauges).

It was shown that the system assessed pressures in the $4.3-8.7 \mathrm{kPa}$ range at the National Metrology Institute at RISE, Sweden with sub-ppm precision $(0.5-0.9 \mathrm{ppm})$. The system was thereafter disassembled, packed and transported $1040 \mathrm{~km}$ to Umeå University, where it, after unpacking and reassembling, demonstrated a similar precision $(0.8-2.1$ ppm). This shows that the system can be disassembled, packed, transported, unpacked, and reassembled with virtually unchanged performance.

The results indicate that this type of system is suited for a variety of applications. For example, it can be used as a travelling standard by which various pressure measuring systems at dissimilar premises can be characterized with respect to each other. It can also be used, in combination with a local primary standard, for calibration of pressure systems, including those that do not have a measurement range that overlap with the local primary standard. It is also possible to use the system for benchmarking or characterization of other refractometry systems with respect to pressure induced cavity deformation and wavelength dependence of refractivity.

Based on this work, several upgrades of the system can be envisioned, both to increase the performance of the system (in particular its accuracy) and to improve on its transportability. Some of these are under implementation. Following these, a more rigorous measurement campaign involving the transportable refractometer is planned, using fully calibrated pressure balances, in the form of a circle comparison involving multiple laboratories, which should provide information about the repeatability and the accuracy of the system.

Parallel to the improvement of the transportable system, a number of actions are presently being pursued to address the accuracy of the technique, e.g.;

\footnotetext{
${ }^{8}$ They can predominantly be attributed to uncertainties in the set values of the pressure balances (possibly by an offset in the pressure gauge assessing the hood pressure).
} 
assessment of the cavity deformation [26]; improved assessment of temperature [24]; and the use of (or assessment of) updated gas coefficients with less uncertainty, parts of which are scheduled to be carried out within the ongoing EMPIR initiative QuantumPascal (No. 18SIB04).

This project (QuantumPascal, 18SIB04) has received funding from the EMPIR programme co-financed by the Participating States and from the European Union's Horizon 2020 research and innovation programme. The authors acknowledge support from the Swedish Research Council (VR) (Project No. 621-2015-04374); the Umeå University Industrial Doctoral School (IDS); the Vinnova Metrology Programme (Project Nos. 2018-04570 and 201905029); and the Kempe Foundations (Project No. 1823, U12).

\section{REFERENCES}

[1] C. R. Tilford, " 3 and a half centuries later - the modern-art of liquid-column manometry", Metrologia, vol. 30, pp. 545-552, 1994.

[2] J. W. Schmidt, K. Jain, A. P. Miiller, W. J. Bowers, D. A. Olson, "Primary pressure standards based on dimensionally characterized piston/cylinder assemblies", Metrologia, vol. 43, pp. 53-59, 2006.

[3] J. H. Hendricks, D. A. Olson, "1-15,000 Pa Absolute mode comparisons between the NIST ultrasonic interferometer manometers and non-rotating force-balanced piston gauges", Measurement, vol. 43, pp. 664-674, 2010.

[4] J. Ricker, J. Hendricks, T. Bock, P. Dominik, T. Kobata, J. Torres, I. Sadkovskaya, "Final report on the key comparison CCM.P-K4.2012 in absolute pressure from $1 \mathrm{~Pa}$ to $10 \mathrm{kPa}$ ", Metrologia, vol. 54, p. 07002, 2017.

[5] P. F. Egan, J. A. Stone, J. H. Hendricks, J. E. Ricker, G. E. Scace, G. F. Strouse, "Performance of a dual Fabry-Perot cavity refractometer", Opt. Lett., vol. 40, pp. 3945-3948, 2015.

[6] K. Jousten, J. Hendricks, D. Barker, K Douglas., S. Eckel, P. Egan, J. Fedchak, J. Flugge, C. Gaiser, D. Olson, J. Ricker, T. Rubin, W. Sabuga, J Scherschligt., R. Schodel, U Sterr., J. Stone, G. Strouse, "Perspectives for a new realization of the pascal by optical methods", Metrologia, vol. 54, pp. S146-S161, 2017.

[7] A. D. Buckingham, C. Graham, "Density dependence of refractivity of gases", P. Roy. Soc. Lond. A Mat., vol. 337, pp. 275-291, 1974.

[8] H. J. Achterman, T. K. Bose, M. Jaeschke, J. M. Starnaud, "Direct determination of the 2nd refractivity virial-coefficient of methane, nitrogen, and 5 of their mixtures", Int. J. Thermophys., vol. 7, pp. 357-366, 1986.

[9] H. J. Achtermann, G. Magnus, T. K. Bose, "Refractivity virial-coefficients of gaseous $\mathrm{CH}_{4}, \mathrm{C}_{2} \mathrm{H}_{4}$, $\mathrm{C}_{2} \mathrm{H}_{6}, \mathrm{CO}_{2}, \mathrm{SF}_{6}, \mathrm{H}_{2}, \mathrm{~N}_{2}, \mathrm{He}$, and $\mathrm{Ar}$ ", J. Chem. Phys., vol. 94, pp. 5669-5684, 1991.

[10] M. Stock, R. Davis, E. de Mirandes, M. J. T. Milton, "The revision of the SI-the result of three decades of progress in metrology", Metrologia, vol. 56, p. 022001, 2019.
[11] M. Andersson, L. Eliasson, L. R. Pendrill, “Compressible Fabry-Perot refractometer”, Appl. Opt., vol. 26, pp. 4835-4840, 1987

[12] H. Fang, P. Juncar, "A new simple compact refractometer applied to measurements of air density fluctuations", Rev. Sci. Instrum., vol. 70, pp. 31603166, 1999.

[13] H. Fang, A. Picard, P. Juncar, "A heterodyne refractometer for air index of refraction and air density measurements", Rev. Sci. Instrum., vol. 73, pp. 1934-1938, 2002.

[14] D. Mari, M. Bergoglio, M. Pisani, M. Zucco, "Dynamic vacuum measurement by an optical interferometric technique", Meas. Sci. Technol., vol. 25, p. 125303, 2014.

[15] L. P. Yan, B. Y. Chen, E. Z. Zhang, S. H. Zhang, Y. Yang, "Precision measurement of refractive index of air based on laser synthetic wavelength interferometry with Edlen equation estimation", Rev. Sci. Instrum., vol. 86, p. 085111, 2015.

[16] P. Egan, J. A. Stone, "Absolute refractometry of dry gas to $+/-3$ parts in $10^{9} "$, Appl. Opt., vol. 50, pp. 3076-3086, 2011.

[17] I. Silander, M. Zelan, O Axner, F. Arrhén, L. Pendrill, A. Foltynowicz, "Optical measurement of the gas number density in a Fabry-Perot cavity", Meas. Sci. Technol., vol. 24, pp. 105207, 2013.

[18] T. Rubin, Y. Yang, "Simulation of pressure induced length change of an optical cavity used for optical pressure standard", Journal of Physics: Conf. Series, vol. 1065, p. 162003, 2018.

[19] R. W. Fox, "Temperature analysis of low-expansion Fabry-Perot cavities", Opt. Express, vol. 17, pp. 15023-15031, 2009.

[20] I Silander, T. Hausmaninger, M. Zelan, O. Axner, "Gas modulation refractometry for high-precision assessment of pressure under non-temperature-stabilized conditions", J. Vac. Sci. Technol. A, vol. 36, p. 03E105, 2018.

[21] M. Zelan, I. Silander, C. Forssén, J. Zakrisson, O. Axner, "Recent advances in Fabry-Perot-based refractometry utilizing gas modulation for assessment of pressure", Acta IMEKO 2020

[22] I. Silander, T. Hausmaninger, C Forssén., M. Zelan, O. Axner, "Gas equilibration gas modulation refractometry for assessment of pressure with subppm precision”, J. Vac. Sci. Technol. B, vol. 37, pp. $042901,2019$.

[23] R. W. P. Drever, J. L. Hall, F. V. Kowalski, J. Hough, G. M. Ford, A. J. Munley, H. Ward, “ Laser phase and frequency stabilization using an optical resonator", Appl. Phys. B, vol. 31, pp. 97-105, 1983.

[24] I. Silander, C. Forssén, J. Zakrisson, M. Zelan, O. Axner, "An Invar-based Fabry-Perot cavity refractometer with a gallium fixed-point cell for assessment of pressure", Acta IMEKO 2020.

[25] I. Silander, C. Forssén, J. Zakrisson, M. Zelan, O. Axner, "Invar-based refractometer for pressure assessments", Opt. Lett., vol. 45, pp. 2652-56, 2020.

[26] J. Zakrisson, I. Silander, C. Forssén, M. Zelan, O. Axner, "Procedure for robust characterization of Fabry Perot based refractometer", J. Vac. Sci. Technol. B, vol 38, 2020. 